\title{
INITIAL FOLLOW-UP OF OPTICAL TRANSIENTS WITH COLORES USING THE BOOTES NETWORK
}

\author{
M. D. Caballero-Garcia ${ }^{a, *}$, M. Jelinek ${ }^{b}$, A. Castro-Tirado $^{b}$, \\ R. Hudec ${ }^{a, c}$, R. Cunniffe ${ }^{b}$, O. Rabaza ${ }^{d}$, L. Sabau-Graziati ${ }^{e}$ \\ ${ }^{a}$ Czech Technical University in Prague, Technická 2, 166 27 Praha 6 (Prague), Czech Republic \\ ${ }^{b}$ Instituto de Astrofísica de Andalucía (IAA-CSIC), P.O. Box 03004, E-18080, Granada, Spain \\ ${ }^{c}$ Astronomical Institute, AS CR, 25165 Ondrejov, Czech Republic \\ d Área de Ingeniería Eléctrica, Dpto. de Ingeniería Civil, Univ. de Granada, Spain \\ e División de Ciencias del Espacio (INTA), Torrejón de Ardoz, Madrid, Spain \\ * corresponding author: cabalma1@fel.cvut.cz
}

\begin{abstract}
The Burst Observer and Optical Transient Exploring System (BOOTES) is a network of telescopes that allows the continuous monitoring of transient astrophysical sources. It was originally devoted to the study of the optical emission from gamma-ray bursts (GRBs) that occur in the Universe. In this paper we show the initial results obtained using the spectrograph COLORES (mounted on BOOTES-2), when observing optical transients (OTs) of diverse nature.
\end{abstract}

KEYwords: Telescopes, gamma-ray burst: general, stars: variables: general .

\section{INTRODUCTION}

The majority of the sources visible in the sky have variable emission. Some of them vary in a long timescale compared to the human life. But most of them have shorter variability and, because of that, are continuously observed by telescopes both on Earth and space. The study of variability provides one of the most direct clues on the size and physical characteristics of the emitting object. In the highest energy regime (X-rays, $\gamma$-rays) the scales are related to orbits around compact objects (i.e., the result from the death of massive stars). For longer wave-lengths variability is related to larger regions and/or bigger stars. The most extreme case is the study of microwaves, that allows the study of large structures in the Universe. This shows that it is important for astrophysicists to continuously observe our changing Universe, from the smallest to the largest scales, for its understanding.

The study of variable sources in the optical regime can be performed from the Earth surface. Furthermore, it is a window for the study of many complex physical processes occurring at intermediate space/time scales (see the previous paragraph). In this regime we can study (variable) stars (e.g., erupting variables, novae, cataclysmic variables, etc.), the most violent physical processes in the Universe (SuperNovae - hereafter SNe - and Gamma-Ray Bursts), the relatively quiet Active Galactic Nuclei (AGN) and Quasars (QSOs) and the outer discs and stellarcompanions around compact objects (neutron stars and black holes). Nevertheless, the window is still open to discoveries of new types of transients. Recently, thanks to the advance of devices and detectors, new phenomena are being studied (gravitational lenses, binary mergers, stellar tidal disruptions by black holes). It is natural to wonder whether there are new (undiscovered) types of physical processes (sources) giving rise to new kinds of observed emission.

Observations in the optical are performed by big and medium-sized telescopes on Earth. The former are not suitable for performing the rapid follow-up needed for the study of optical transients (as we will explain hereafter). These transients events are typically of short duration (from fractions of a second to a few days), because the physical processes that originate them are of limited duration/spatial extent. Robotic smaller telescopes are very well suited for performing such studies. This is due to several factors: their observing flexibility, their rapid response and slew times and the fact that they can be located worldwide working remotely (therefore allowing continuous monitoring). Of course, additional observations might be triggered after the transient has been detected with large X-ray/Optical Observatories. In this way we can perform deep studies on the nature of these sources.

\subsection{The Burst Optical Observer and Transient Exploring System AND ITS SPECTROGRAPHS}

BOOTES (acronym of the Burst Observer and Optical Transient Exploring System) is a world-wide network of robotic telescopes. It was originally designed from a Spanish-Czech collaboration that started in 1998 [1, 2]. The telescopes are located at Huelva (BOOTES-1), Malaga (BOOTES-2), Granada, Auckland (BOOTES3) and Yunnan (BOOTES-4), located at Spain, New Zealand and China, respectively. There are plans of extending this network even further (Mexico, South Africa, Chile, etc.). These telescopes are medium- 
sized ( $\mathrm{D}=30-60 \mathrm{~cm}$ ), autonomous and very versatile. They are very well suited for the continuous study of the fast variability from sources of astrophysical origin (Gamma-Ray Bursts - hereafter GRBs, and Optical Transients - hereafter OTs).

Currently two spectrographs are built and working properly in the network at Malaga and Granada (in the optical and infra-red, respectively). In Section 2 we will show preliminary results obtained so far with COLORES at BOOTES-2 in the field of OTs of astrophysical origin.

\subsection{COLORES}

COLORES stands for Compact Low Resolution Spectrograph [3]. It is a spectrograph designed to be lightweight enough to be carried by the high-speed robotic telescope $60 \mathrm{~cm}$ (BOOTES-2). It works in the wavelength range of $3800-11500 \AA$ and has a spectral resolution of $15-60 \AA$. The primary scientific target of the spectrograph is a prompt GRB follow-up, particularly the estimation of redshift.

COLORES is a multi-mode instrument that can switch from imaging a field (target selection and precise pointing) to spectroscopy by rotating wheelmounted grisms, slits and filters. The filters and the grisms (only one is mounted at the moment) are located in standard filter wheels and the optical design is comprised of a four-element refractive collimator and an identical four-element refractive camera. As a spectroscope, the instrument can use different slits to match the atmospheric seeing, and different grisms in order to select the spectral resolution according to the need of the observation.

The current detector is a $1024 \times 1024$-pixel device, with 13-micron pixels. The telescope is a rapid and lightweight design, and a low instrument weight was a significant constraint in the design as well as the need to be automatic and autonomous. For further details on description, operation and working with COLORES we refer to M. Jelinek's PhD thesis and references therein.

\subsection{SCIENTIFIC GOALS}

The BOOTES scientific goals are multifold, and are detailed in the following.

Observations of GRB optical counterparts. There have been several GRBs for which the optical counterpart has been detected simultaneously to the gamma-ray event, with magnitudes in the range 510th. These observations provide important results on the central engine of these sources. The fast slewing $0.6 \mathrm{~cm}$ BOOTES telescopes are providing important results in this field [4].

The detection of OTs of cosmic origin. These events could be unrelated to GRBs and could constitute a new type of astrophysical phenomenon (perhaps associated to QSOs/AGN). If some of them are related to GRBs, the most recent GRB models predict that here should be a large number of bursting sources in which only transient X-ray/optical emission should be observed, but no gamma-ray emission. The latter would be confined a jet-like structure and pointing towards us only in a few cases.

\section{Monitoring a range of astronomical objects.} These are astrophysical objects ranging from galactic sources such as comets, cataclysmic variables, recurrent novae, compact objects in X-ray binaries to extragalactic sources such as distant $\mathrm{SNe}$ and AGN. In the latter case, there are hints that sudden and rapid flares occur.

\section{SCIENTIFIC RESULTS USING BOOTES-2/COLORES}

In the following we present some important scientific results obtained using BOOTES-2 and its lowresolution spectrograph COLORES, since the beginning of its operation (Summer of 2012).

\subsection{GRB 130606A}

Since the first light on 1998, more than a hundred of GRBs have been observed with BOOTES, some of them only $\approx 30$ s after the onset of the gamma-ray event. The majority of the results have been published in circulars and refereed journals 1 . Here we focus on the recent event occurred on 6th June 2013.

$\mathrm{A} \approx 275$ s cosmic gamma-ray burst (GRB 130606A) was recorded by Swift and KONUS-Wind on 6th June 2013, 21:04:34 U.T. (T0) [5, 6] displaying a bright afterglow (the emission at other wavelengths following the gamma-rays) in X-rays, but no apparent optical transient emission [7] in the range of the UVOT telescope aboard Swift. BOOTES-2 station automatically responded to the alert and an optical counterpart was identified [8], thanks to the spectral response of the detector up to $1 \mu \mathrm{m}$, longer than that of Swift/UVOT $(0.17-0.65 \mu \mathrm{m})$. We refer to [9] for details on the observations and results.

\subsection{TCP J17154683-3128303 = NOVA SCORPIUS 2014}

Following the discovery on 26th March 2014 of a $10 \mathrm{mag}$ new source in Scorpius dubbed TCP J17154683-3128303 by [10] also detected by Swift on March 27 [11, [12] report that an optical spectrum was obtained with the COLORES spectrograph at the $0.6 \mathrm{~m}$ robotic telescope at the BOOTES- 2 astronomical station (see Figure 1). The spectrum, covering the range $3800-200 \AA$ has been taken on $30^{\text {th }}$ March, 04:37 UT and shows broad emission lines of Balmer series, He I 501.6, 587.8, 706.5, and probably of O I 844.6, suggesting a nova in early phase (Nova Scorpius 2014) thus confirming the earlier suggestion by [13].

\footnotetext{
${ }^{1}$ We refer to http://bootes.iaa.es for further information on the BOOTES network and scientific results.
} 


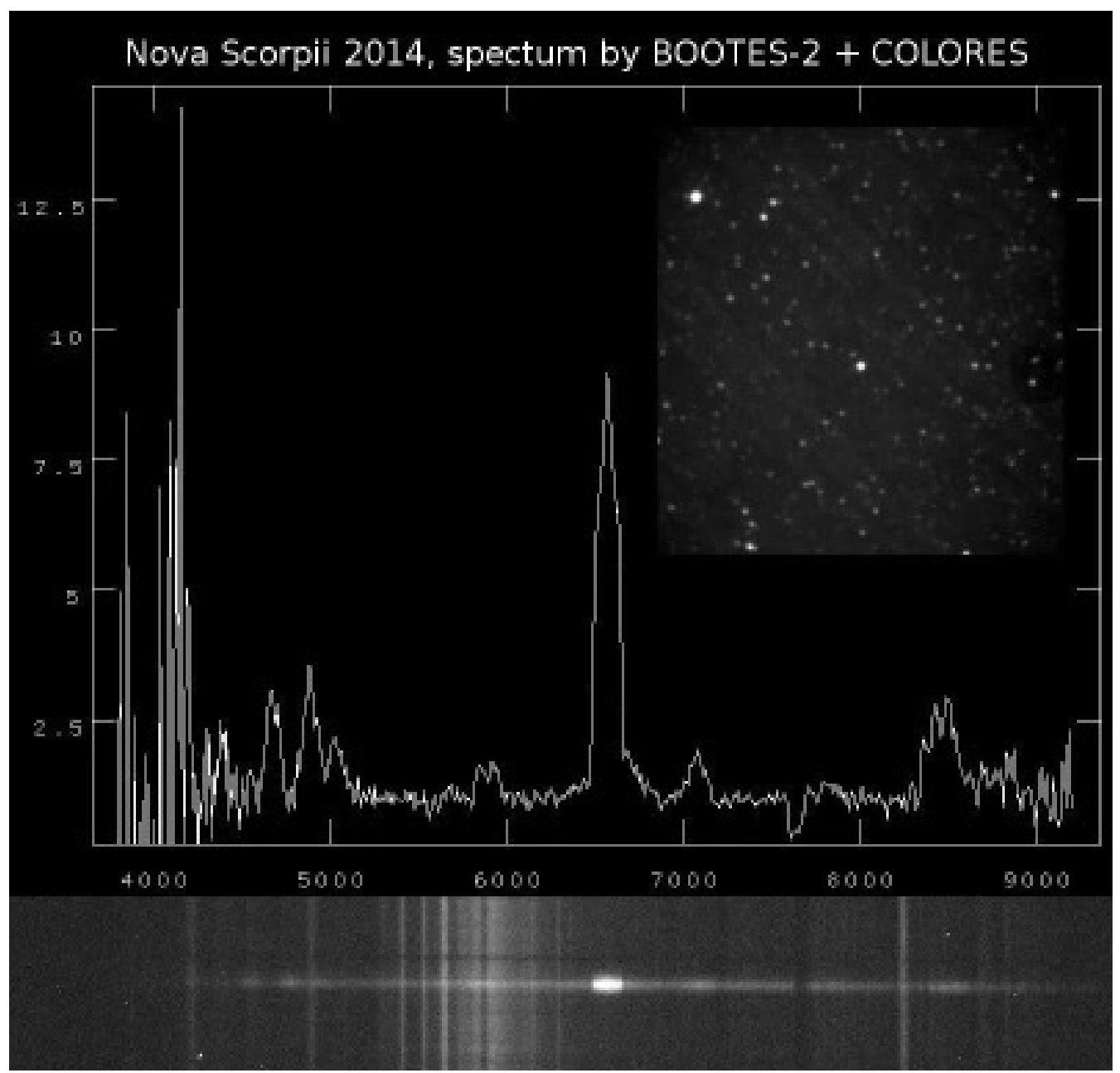

FigURE 1. Optical spectrum in the range 3800-9200 ̊ from Nova Scorpii 2014, obtained with BOOTES-2/COLORES on 30th March, 04:37 UT.

\subsection{OPTICAL SPECTRA FROM RED-DWARF FLARING STARS}

The Swift team reported on the detection of a superflare from one of the stars in the close visual $\mathrm{dM} 4 \mathrm{e}+\mathrm{dM} 4 \mathrm{e}$ flare star binary system DG CVn. The Burst Alert Telescope (BAT) triggered on DG CVn the 23rd April 2014 at 21:07:08 UT (T0) 14]. BOOTES2/COLORES has been observing DG CVn since the beginning of the superflare and new interesting optical spectral variability following the X-ray superflare evolution has been observed (we refer to [15] for further details).

Additionally, following the detection and subsequent monitoring of the new outburst from the RS CVn UX Ari by SWIFT and MAXI [17, 18, the $0.6 \mathrm{~m}$ robotic telescope at the BOOTES-2 astronomical station, obtained optical 4000-9000 ̊ spectra starting at 19th July 2014, 01:32:24.382 UT and ending at 04:25:55.652 UT. It was reported [16] that the optical spectra contain broad molecular $\mathrm{TiO}, \mathrm{CaI}, \mathrm{MgI}, \mathrm{NaI}$ lines plus a red continuum (see Figure 2). These spectra lack of any significant Balmer lines in emission. These spectral features are indicative of a late-type star spectrum (as previously reported). Nevertheless, there are no indications of important chromospheric activity, that might have been disappeared by the time of our observations.

\section{DisCUSSION AND CONCLUSIONS}

The era of OTs is about to start. Since the beginning of the modern times the big telescopes have been the only resource for astronomers to study astrophysical sources. In spite of constituting the best tool for deep studies of individual targets, they are not properly suited for the discovery of optical transient sources. Their big size limits the speed they can cover the entire sky and the time for overheads might be longer than that for medium-sized telescopes. Many factors make them difficult to fully automatize and indeed currently none is completely robotic and autonomous. Mediumsized telescopes (i.e., $\mathrm{D} \leq 1 \mathrm{~m}$ ) can be much quicker moving from target to target and time overheads are usually very small. Therefore, robotic medium-sized telescopes are currently the best ones for the followup and studies of the long-term variability of the astrophysical transient sources.

BOOTES-2 constitutes one step forward with respect to any robotic telescope on Earth that has ex- 


\section{UX Ari, spectrum by BOOTES-2 + COLORES}

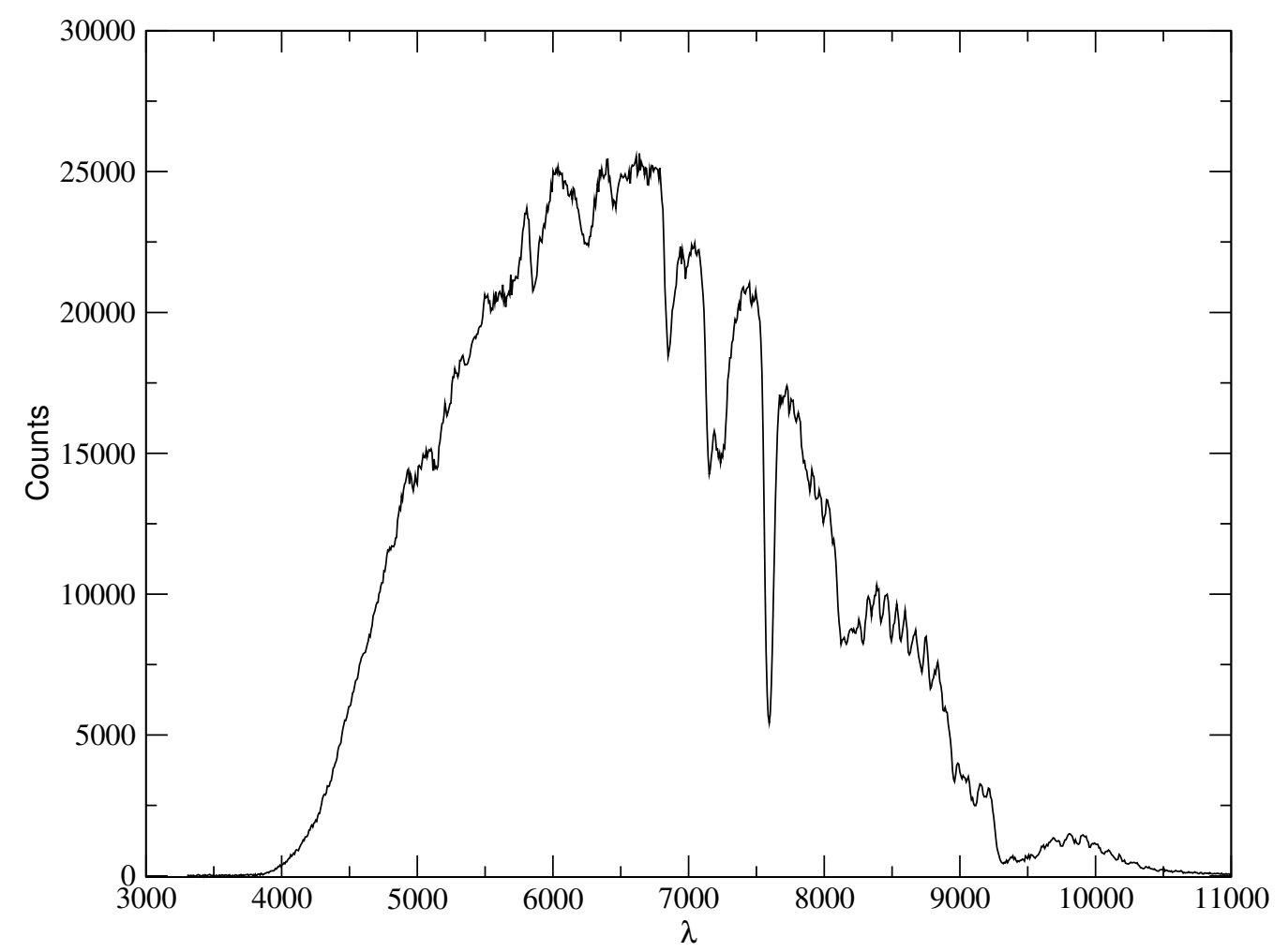

FiguRE 2. Optical spectrum in the range 3800-11500 $\AA$ from UX Ari, obtained with BOOTES-2/COLORES on 19th July, 01:32 UT.

isted so far. This is because it is the first robotic telescope with a spectrograph mounted on it which has demonstrated to work properly. This does not allow only to perform early follow-up and to measure the redshift of GRBs, with cosmic origin, but also to perform early follow-up (both photometric and spectroscopic) of transient sources, often located much more nearby. These OTs might have been reported at other wave-lengths (typically at X-and $\gamma$-rays), which would create an alert to the scientific community (often through an Astronomer Telegram). In such a case the observer (remotely) sends the telecommands to start the observation with BOOTES-2, that re-observes the target every time it is visible during the following nights.

Apart from the intensive campaign of follow-up of GRBs performed by the BOOTES network $(\approx 100$ GRBs have been observed so far), BOOTES-2 and its spectrograph (COLORES) are providing excellent results in the field of OTs, too. In this paper we mention a few of them, obtained during the last $1.5 \mathrm{yr}$ (since the spectrograph was mounted on the telescope). But this is only the beginning and we look forward to follow many OTs for understanding better their physical properties and may be also to discover and follow-up new kinds of OTs. This will prepare us for the advent of the Large Synoptic Survey Telescope (LSST), the biggest telescope ever built on Earth for the study of the entire sky, that will allow the discovery of many kinds of new astrophysical sources (if they exist) and follow-up of OTs, planned to start operations on 2023.

\section{ACKNOWLEDGEMENTS}

MCG is supported by the European social fund within the framework of realizing the project "Support of inter-sectoral mobility and quality enhancement of research teams at Czech Technical University in Prague", CZ.1.07/2.3.00/30.0034. MJ and AJCT thank the support of the Spanish Ministry Projects AYA2009-14000-C03-01 and AYA2012-39727-C03-01. RH acknowledges GACR grant 13-33324S.

\section{REFERENCES}

[1] Castro-Tirado, A. J., Soldán, J., Bernas, M. et al. The Burst Observer and Optical Transient Exploring System (BOOTES). AEAS, 138, 583, 1999.

[2] Castro-Tirado, A. J., Jelínek, M., Gorosabel, J. et al. Building the BOOTES world-wide Network of Robotic telescopes. ASI Conf. Ser. 7, 313, 2012.

[3] Rabaza, O., Jelinek M., Castro-Tirado A. J., et al. Compact low resolution spectrograph (COLORES), an imaging and long slit spectrograph for robotic telescopes. Review of Scientific Instruments 84 (11), 114501, 2014.

[4] Jelínek, M., Castro-Tirado, A. J., de Ugarte Postigo, A., Kubánek, P. et al. Four Years of Real-Time GRB Followup by BOOTES-1B (2005-2008). Advances in Astronomy, 85, 2010. 
[5] Barthelmy, S. D., Baumgartner, W. H., Cummings, J. R., et al. GRB 130606A, Swift-BAT refined analysis. GRB Coordinates Network, 14819, 2013.

[6] Golenetskii, S., Aptekar, R., Mazets, E. et al. Konus-wind observation of GRB 130606A. GRB Coordinates Network, 14808, 2013.

[7] Ukwatta, T. N. and Barthelmy, S. D. and Gehrels, N. et al. GRB 130606A: Swift detection of a burst. GRB Coordinates Network, 14781, 2013.

[8] Jelinek, M., Gorosabel, J., Castro-Tirado, A. J. et al. GRB 180606A: optical afterglow with BOOTES-2/TELMA and 1.23m CAHA. GRB Coordinates Network, 14782, 2013.

[9] Castro-Tirado, A. J., Sánchez-Ramírez, R., Ellison, S. L. et al. GRB 130606 A within a sub-DLA at redshift 5.91. Submitted to AEAA, astro-ph/1312.5631, 2013.

[10] Nishiyama, K. \& Kabashima, F. CBAT Transient Object Follow-up Reports.

[11] Kuulkers, E., Page, K. L., Saxton, R. D., Ness, J.-U., et al. Detection of absorbed X-ray emission from TCP J17154683-3128303 by Swift. The Astronomer's Telegram, 6015, 2014.

[12] Jelinek, M., Cunniffe, R., Castro-Tirado, A. J., Rabaza, O., et al. TCP J17154683-3128303 = Nova Scorpius 2014. The Astronomer's Telegram, 6025, 2014.

[13] Noguchi, T., Kojima, T., Pearce, A., et al. Nova Scorpii $2014=$ Tcp J17154683-3128303. Central Bureau Electronic Telegrams, 3841, 2014.

[14] D'Elia, V., Gehrels, N., Holland, S. T. et al. Swift Trigger 596958 is probably the flare star: DG CVn. GRB Coordinates Network, 16158, 2014.

[15] Caballero-Garcia, M. D., et al. In preparation, 2014.

[16] Caballero-Garcia, M. D., Castro-Tirado, A. J., Jelinek, M., on behalf of a larger collaboration. Optical spectra of UX Ari with BOOTES-2. The Astronomer's Telegram, 6337, 2014.

[17] Kawagoe, A., Tsuboi, Y., Negoro, H. et al. MAXI/GSC detection of a big flare from UX Ari. The Astronomer's Telegram, 6315, 2014.

[18] Krimm, H. A., Drake, S. A., Hagen, L. M. Z. et al. Swift observations of a flare from UX Ari. The Astronomer's Telegram, 6319, 2014. 